\title{
Meson production in high-energy electron-nucleus scattering
}

\author{
Göran Fäldt* \\ Department of physics and astronomy, \\ Uppsala University, Box 516, S-751 20 Uppsala,Sweden
}

(Dated: November 11, 2018)

\begin{abstract}
Pseudoscalar mesons can be produced and studied in high-energy electron-nucleus scattering. We review and extend our previous analysis of meson production in the nuclear Coulomb field. The $P \rightarrow \gamma \gamma$ decay rates are most directly determined for mesons produced in the double-Coulomb region where both photons are nearly real, and provided the background-hadronic contribution remains small. The larger the mass of the meson the higher the electron energy needed to assure such a condition.
\end{abstract}

PACS numbers: 24.10.Ht, 25.20.Lj, 25.30.Rw

*Electronic address: goran.faldt@physics.uu.se 


\section{INTRODUCTION}

Pseudoscalar mesons are produced in high-energy electron-nucleus scattering through a two-photon process where the two photons are radiated, one by the high-energy electron and the other by the atomic nucleus. This possibility was studied, in the Born approximation, by Hadjimichael and Fallieros [1]. Recently, a Glauber-model [2] description of the same process

has been developed [3]. Theoretical studies of the two-photon-fusion process are particularly valuable since the PrimEx Collaboration [4] aims at measuring electromagnetic properties of pseudoscalar mesons through this effect, in $11 \mathrm{GeV} / c$ electron-nucleus scattering.

The kinematics of the electron-nucleus-meson-production reaction is defined through

$$
e^{-}\left(k_{1}\right)+\mathrm{A}\left(p_{1}\right) \rightarrow e^{-}\left(k_{2}\right)+P(K)+A\left(p_{2}\right),
$$

where $P$ represents one of the mesons $\pi^{0}, \eta$, or $\eta^{\prime}$. Our analysis is relevant for high electron and meson energies and small transverse momenta. In addition, the momentum transfers to meson and nucleus should preferentially be in the double-Coulomb region, leading to further restrictions. The mechanism dominating this region is, at sufficiently high energies, bescribed by the graph of Fig.1.

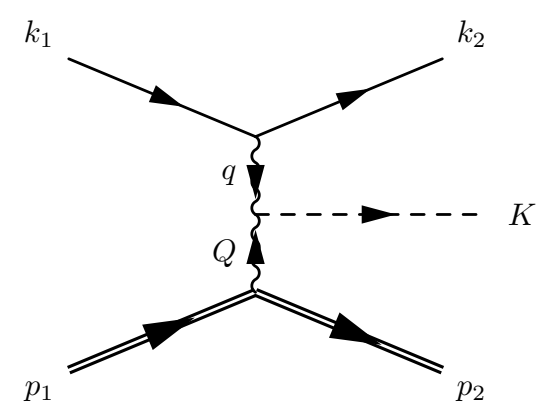

FIG. 1: Graph describing pseudoscalar-meson production in the Coulomb field of a nucleus in electron coherent-nucleus scattering.

This graph shows that pion-nucleus photoproduction is a subgraph of the pion-nucleus electroproduction, but only in special kinematic circumstancies does the photoproduction amplitude enter as a separate factor. Out treatment of the electroproduction amplitude is similar to our previous treatment of the photoproduction amplitude [5].

The cross-section distribution is in the Coulomb region mainly determined by the photon 
propagators, which lead to structures like

$$
\frac{k_{\perp}}{\mathbf{k}_{\perp}^{2}+k_{\|}^{2} / \gamma^{2}}
$$

with $\mathbf{k}_{\perp}$ the variable photon transverse momentum, $k_{\|}$the fixed photon longitudinal momentum, and $\gamma$ the gamma factor of the radiating charge. This behaviour results in a cross-section maximum at $k_{\perp}=k_{\|} / \gamma$, the Primakoff peak. For the low-energy photon radiated by the nucleus $k_{\|} / \gamma=m_{P}^{2} / 2 K_{\|}$, where $m_{P}$ is the mass of the meson and $K_{\|}=\mathbf{K} \cdot \hat{k}_{1}$ its longitudinal (or total) momentum. This expression is well known from ordinary Coulomb production. For the high-energy photon radiated by the high energy electron the effective longitudinal momentum is $k_{\|} / \gamma=m_{e}$, with $m_{e}$ the electron mass.

In the PrimEx experiment [4] typical energies are; for the incident electron $E\left(\mathbf{k}_{1}\right)=11$ $\mathrm{GeV}$, for the scattered electron $E\left(\mathbf{k}_{2}\right)=300 \mathrm{MeV}$, and for the pseudoscalar meson $\omega(\mathbf{K})=$ 10.7 GeV. Consequently, the energy of the virtual photon radiated by the electron, and initiating the meson production through fusion with a soft photon radiated by the nucleus, is also $10.7 \mathrm{GeV}$. These numbers are only for numerical illustration. The model we present is in itself a general one.

The electron-coherent-nucleus-production amplitude is a sum of two amplitudes; the twophoton-fusion amplitude $\mathcal{M}_{2 \gamma}$ of Fig.1, and the electron induced hadronic-photoproduction amplitude $\mathcal{M}_{\gamma}$ of Fig.2.

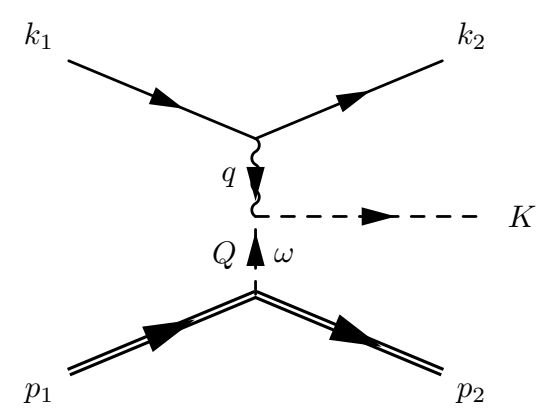

FIG. 2: Graph describing the hadronic contribution to pseudoscalar-meson product in electron coherent-nucleus scattering. Dominant contribution from $\omega$ exchange.

Normalizations are chosen such that in the Born approximation the unpolarized crosssection distribution for the two-photon-fusion contribution simplifies to

$$
\frac{\mathrm{d} \sigma}{\mathrm{d}^{2} k_{2 \perp} \mathrm{d}^{2} K_{\perp} \mathrm{d} k_{2 \|}}=\frac{1}{\pi K_{\|}}\left[\frac{Z \alpha^{2}}{m_{\pi}} g_{\pi \gamma \gamma} \frac{q_{\perp}}{\mathbf{q}_{\perp}^{2}+m_{e}^{2}} \cdot \frac{Q_{\perp}}{\mathbf{Q}_{\perp}^{2}+Q_{\|}^{2}}\right]^{2},
$$


with $-Q_{\|}=m_{P}^{2} / 2 K_{\|}$. The structure of the cross-section distribution at small-transverse momenta is essentially determined by the photon-exchange propagators. There are two such propagators; one in the variable $\boldsymbol{q}_{\perp}$ and one in the variable $\boldsymbol{Q}_{\perp}$. Each of them exhibits a Primakoff-peak structure. We observe that the spin-averaged Born approximation does not depend on the angle between the transverse momenta $\mathbf{q}_{\perp}$ and $\mathbf{Q}_{\perp}$.

The numerical illustrations in Ref.[3] erroneously employ positive values for $Q_{\|}$. However, this error is of no concern since the quantities graphed are independent of the sign of $Q_{\|}$. 


\section{CLASSICAL RADIATION BY A RELATIVISTIC ELECTRON}

The pseudoscalar-meson decay $P \rightarrow \gamma \gamma$ is governed by the Hamiltonian density

$$
\mathcal{H}(x)=\frac{1}{8} g F(x) \cdot \tilde{F}(x) P(x),
$$

where $F_{\mu \nu}(x)$ is the electromagnetic-field tensor, $\tilde{F}_{\mu \nu}(x)=\epsilon_{\mu \nu \sigma \tau} F^{\sigma \tau}$ its dual, with $\epsilon_{0123}=1$, and $P(x)$ the pseudoscalar-meson field. The coupling constant $g=e^{2} g_{\pi \gamma \gamma} / m_{\pi}$. In terms of the electric and magnetic fields the Hamiltonian reads

$$
\mathcal{H}(x)=\frac{1}{2} g \mathbf{E}(x) \cdot \mathbf{B}(x) P(x) .
$$

In our application the electromagnetic fields are generated by the electron and nuclear charges. It is therefore instructive to study first the classical counterparts of these fields [6].

A point particle of charge $e$ moves along the trajectory $\mathbf{r}(t)=\mathbf{r}_{0}+\mathbf{v} t$. The associated fourvector potential $A_{\mu}(x)$ satisfies the Maxwell equation $\square A(x)=j(x)$, with the four-vector current

$$
j(x)=e(1, \mathbf{v}) \delta\left(\mathbf{r}-\mathbf{r}_{0}-\mathbf{v} t\right) .
$$

In a plane-wave decomposition of the four-vector potential,

$$
A(x)=\int \frac{\mathrm{d}^{3} k}{(2 \pi)^{3}} A(\mathbf{k}, t) e^{i \mathbf{k} \cdot \mathbf{r}},
$$

the solution to the Maxwell equations takes the form

$$
A(\mathbf{k}, t)=e(1, \mathbf{v}) \frac{e^{-i \mathbf{k} \cdot\left(\mathbf{r}_{0}+\mathbf{v} t\right)}}{\mathbf{k}^{2}-(\mathbf{k} \cdot \mathbf{v})^{2}} .
$$

This four-vector potential gives the following plane-wave decompositions of the electric and magnetic fields

$$
\begin{aligned}
\mathbf{E}(\mathbf{k}, t) & =i e \frac{-\mathbf{k}+(\mathbf{k} \cdot \mathbf{v}) \mathbf{v}}{\mathbf{k}^{2}-(\mathbf{k} \cdot \mathbf{v})^{2}} e^{-i \mathbf{k} \cdot\left(\mathbf{r}_{0}+\mathbf{v} t\right) \mathbf{v} t}, \\
\mathbf{B}(\mathbf{k}, t) & =i e \frac{\mathbf{k} \times \mathbf{v}}{\mathbf{k}^{2}-(\mathbf{k} \cdot \mathbf{v})^{2}} e^{-i \mathbf{k} \cdot\left(\mathbf{r}_{0}+\mathbf{v} t\right)}
\end{aligned}
$$

The denominators can be rewritten as

$$
\mathbf{k}^{2}-(\mathbf{k} \cdot \mathbf{v})^{2}=\mathbf{k}_{\perp}^{2}+k_{\|}^{2} / \gamma^{2}
$$


with $\gamma=E / m$ the relativistic gamma factor of the charged particle. Transverse and parallel refer to directions orthogonal and parallel to the velocity $\mathbf{v}$ of the charged particle. The numerators, on the other hand, can be rewritten as

$$
\begin{aligned}
\mathbf{k}-(\mathbf{k} \cdot \mathbf{v}) \mathbf{v} & =\left(\mathbf{k}_{\perp}, k_{\|} / \gamma^{2}\right), \\
\mathbf{k} \times \mathbf{v} & =\mathbf{k}_{\perp} \times \mathbf{v} .
\end{aligned}
$$

In the application to the PrimEx experiment two limits are of interest. The nonrelativistic limit, $v \ll 1$, applies to the soft radiation by the nucleus. This corresponds to cyclotron radiation. The denominators of Eqs (9) and (10) reduce to $\mathbf{k}^{2}$. The other limit applies to hard radiation by the ultra-relativistic electron, where, in the denominator, the longitudinal momentum $k_{\|}$is cut down by the Lorentz factor $1 / \gamma$. This corresponds to synchrotron radiation.

In the PrimEx experiment there are kinematic restrictions. The longitudinal momentum of the soft photon is fixed to be $m_{P}^{2} / 2 K_{\|}$, with $K_{\|}$the pion momentum, and the longitudinal momentum of the hard photon is very nearly equal to the momentum of the electron, so that $k_{\|} / \gamma \approx m_{e}$. Thus, these two parameters act as cut-offs for the corresponding transverse momentum distributions. The longitudinal component of the electric field is for the hard photon cut down by the factor $1 / \gamma^{2}$ and may be neglected.

According to Eq.(5) pion decay is determined by the scalar product $\mathbf{E}(x) \cdot \mathbf{B}(x)$. The electric field must be associated with the non-relativistic nucleus, since its magnetic field is very weak, and the magnetic field consequently associated with the ultra-relativistic electron. 


\section{THE COULOMB CONTRIBUTION}

We shall now review the formulae for the Coulomb contribution to electron-induced pion production, i.e. the two-photon-fusion amplitude of Fig. 1. The corresponding cross-section distribution has a double-peak-Primakoff structure. We normalize so that the cross-section distribution takes the form

$$
\frac{\mathrm{d} \sigma}{\mathrm{d}^{2} k_{2 \perp} \mathrm{d}^{2} K_{\perp} \mathrm{d} k_{2 \|}}=\frac{1}{\pi K_{\|}}\left[u^{\dagger}\left(k_{2}\right) \mathcal{M}_{2 \gamma} u\left(k_{1}\right)\right]^{2}
$$

where $u\left(k_{1}\right)$ and $u\left(k_{2}\right)$ are two-component spinors for incident and scattered electrons. The two-photon amplitude is decomposed as

$$
\begin{aligned}
\mathcal{M}_{2 \gamma} & =i \mathcal{N}_{2 \gamma}\left[G(\mathbf{q}, \mathbf{Q})-H(\mathbf{q}, \mathbf{Q}) i \boldsymbol{\sigma} \cdot \hat{k}_{1}\right] \\
\mathcal{N}_{2 \gamma} & =Z \frac{\alpha^{2} g_{\pi \gamma \gamma}}{m_{\pi}}
\end{aligned}
$$

The amplitude $G(\mathbf{q}, \mathbf{Q})$ originates with the current part of the electron-four-vector current, and $H(\mathbf{q}, \mathbf{Q})$ with the spin part of the same current.

Taking into account multiple scattering of both electron and meson the expressions for the amplitudes become

$$
\begin{aligned}
G(\mathbf{q}, \mathbf{Q})= & \int \mathrm{d}^{3} r_{e} \int \mathrm{d}^{3} r_{\pi} e^{i \mathbf{q} \cdot\left(\mathbf{r}_{e}-\mathbf{r}_{\pi}\right)} e^{-i \mathbf{Q} \cdot \mathbf{r}_{\pi}} \exp \left[i \chi_{C}\left(b_{e}\right)-\frac{1}{2} \sigma_{\pi}^{\prime} T\left(\mathbf{b}_{\pi}, z_{\pi}\right)\right] \\
& \times\left[\mathbf{E}_{e}\left(\mathbf{r}_{e}-\mathbf{r}_{\pi}\right) \times \mathbf{E}_{A}\left(\mathbf{r}_{\pi}\right)\right] \cdot \hat{k}_{1}, \\
H(\mathbf{q}, \mathbf{Q})= & \int \mathrm{d}^{3} r_{e} \int \mathrm{d}^{3} r_{\pi} e^{i \mathbf{q} \cdot\left(\mathbf{r}_{e}-\mathbf{r}_{\pi}\right)} e^{-i \mathbf{Q} \cdot \mathbf{r}_{\pi}} \exp \left[i \chi_{C}\left(b_{e}\right)-\frac{1}{2} \sigma_{\pi}^{\prime} T\left(\mathbf{b}_{\pi}, z_{\pi}\right)\right] \\
& \times\left[\mathbf{E}_{e}\left(\mathbf{r}_{e}-\mathbf{r}_{\pi}\right) \cdot \mathbf{E}_{A}\left(\mathbf{r}_{\pi}\right)\right],
\end{aligned}
$$

where $\mathbf{r}_{e}$ and $\mathbf{r}_{\pi}$ are the electron and pion coordinates. The nuclear $\mathbf{E}_{A}(\mathbf{r})$ and electron $\mathbf{E}_{e}(\mathbf{r})$ electric fields are the transverse parts of the corresponding fields. The distortion of the electron-wave function is described by the Coulomb-phase function $\chi_{C}\left(b_{e}\right)$; the distortion of the pion-wave function by the nuclear-thickness function $T\left(\mathbf{b}_{\pi}, z_{\pi}\right)$.

In the $G(\mathbf{q}, \mathbf{Q})$ amplitude of Eq.(17) we recognize $\mathbf{E}_{A}\left(\mathbf{r}_{\pi}\right)$ as the nucleus-electric field, and $\mathbf{E}_{e}\left(\mathbf{r}_{e}-\mathbf{r}_{\pi}\right) \times \hat{k}_{1}$ as the electron-magnetic field, all in accordance with Eq.(15). The $H(\mathbf{q}, \mathbf{Q})$ amplitude of Eq.(18) is associated with the spin current, and its parity is therefore opposite to that of the $G(\mathbf{q}, \mathbf{Q})$ amplitude. Hence, it is built on the scalar product of the two electric fields. 
Next, we give the definitions of the various functions entering the integrands of Eqs (17) and (18). The transverse-electric field of the electron is

$$
\mathbf{E}_{e}(\mathbf{r})=\frac{1}{4 \pi i} \frac{\gamma \mathbf{b}}{\left[\mathbf{b}^{2}+\gamma^{2} z^{2}\right]^{3 / 2}}
$$

The $\gamma$ dependence in this expression, which is in coordinate space, is compatible with the $\gamma$ dependence in momentum space, Eq.(11). I apologize for the unconventional multiplicative constant. The transverse-electric field of the nucleus is in general associated with an extended-charge distribution $\hat{\rho}_{c h}(r)$, which we normalize to unity,

$$
\begin{aligned}
\mathbf{E}_{A}(\mathbf{r}) & =\frac{-Q(r)}{4 \pi i} \frac{\mathbf{b}}{\left[\mathbf{b}^{2}+z^{2}\right]^{3 / 2}}, \\
Q(r) & =4 \pi \int_{0}^{r} \mathrm{~d} r^{\prime} r^{\prime 2} \hat{\rho}_{c h}\left(r^{\prime}\right),
\end{aligned}
$$

and $Q(\infty)=1$. For a uniform-charge distribution with radius $R_{u}$

$$
Q(r)=\left\{\begin{array}{l}
1, r>R_{u} \\
\left(r / R_{u}\right)^{3}, r<R_{u}
\end{array}\right.
$$

Note that the fields of Eqs (19) and (20) are defined to have opposite signs.

The expression for the Coulomb-phase function $\chi_{C}(b)$ of in Eqs (17) and (18) has been given by Glauber [2, 7]. We employ the integral representation

$$
\chi_{C}(b)=-2 \frac{Z \alpha}{v} \int \mathrm{d}^{3} r^{\prime} \hat{\rho}_{c h}\left(r^{\prime}\right)\left[\ln \left(\frac{\mathbf{b}-\mathbf{b}^{\prime}}{2 a}\right)\right],
$$

where $a$ is a cut-off parameter common to all amplitudes. For a point-charge distribution we conclude that

$$
\chi_{p c}(b)=-2 \frac{Z \alpha}{v} \ln (b / 2 a) .
$$

Additional information on the Coulomb-phase function can be found in Ref.[7], in particular expressions for the phase function for uniform-charge distributions.

The pion distortion in Eqs (17) and (18) is controlled by the parameter $\sigma_{\pi}^{\prime}=\sigma_{\pi}\left(1-i \alpha_{\pi}\right)$ with $\sigma_{\pi}$ the pion-nucleon-total-cross section. The target-thickness function $T(\mathbf{b}, z)$ is defined as the integral along the pion trajectory of the nuclear-hadronic-matter density $A \hat{\rho}_{h d}(r)$ as seen by the pion,

$$
T(\mathbf{b}, z)=A \int_{z}^{\infty} \mathrm{d} z^{\prime} \hat{\rho}_{h d}\left(\mathbf{b}, z^{\prime}\right),
$$

for a pion produced at $(\mathbf{b}, z)$. The density $\hat{\rho}_{h d}(\mathbf{r})$ is normalized to unity. 
The amplitudes $G(\mathbf{q}, \mathbf{Q})$ and $H(\mathbf{q}, \mathbf{Q})$ can be written on a more convenient form by making use of the operator replacements, $\boldsymbol{b}_{e} \rightarrow-i \boldsymbol{\nabla}_{q}$ and $\boldsymbol{b}_{\pi} \rightarrow+i \boldsymbol{\nabla}_{Q}$, as described in Ref.[3]. This operation leads to the decomposition

$$
\begin{aligned}
& G(\mathbf{q}, \mathbf{Q})=K(\mathbf{q}, \mathbf{Q})\left(\hat{\mathbf{q}}_{\perp} \times \hat{\mathbf{Q}}_{\perp}\right) \cdot \hat{k}_{1}+L(\mathbf{q}, \mathbf{Q}) \hat{\mathbf{q}}_{\perp} \cdot \hat{\mathbf{Q}}_{\perp} \\
& H(\mathbf{q}, \mathbf{Q})=K(\mathbf{q}, \mathbf{Q}) \hat{\mathbf{q}}_{\perp} \cdot \hat{\mathbf{Q}}_{\perp}-L(\mathbf{q}, \mathbf{Q})\left(\hat{\mathbf{q}}_{\perp} \times \hat{\mathbf{Q}}_{\perp}\right) \cdot \hat{k}_{1} .
\end{aligned}
$$

The expressions for the scalar amplitudes $K(\mathbf{q}, \mathbf{Q})$ and $L(\mathbf{q}, \mathbf{Q})$ are, with $q_{\|} / \gamma \approx m_{e}$,

$$
\begin{aligned}
K(\mathbf{q}, \mathbf{Q})= & \frac{m_{e} Q_{\|}}{(2 \pi)^{2}} \int_{0}^{\infty} b_{e} \mathrm{~d} b_{e} K_{1}\left(m_{e} b_{e}\right) \int_{0}^{\infty} b_{\pi} \mathrm{d} b_{\pi} I\left(b_{\pi}, Q_{\|}\right) \\
& \times 2 \pi \int_{0}^{2 \pi} \mathrm{d} \phi J_{0}(\sqrt{X})\{\cos \phi\} \exp \left[i \chi_{C}\left(B_{e}\right],\right. \\
L(\mathbf{q}, \mathbf{Q})= & \frac{m_{e} Q_{\|}}{(2 \pi)^{2}} \int_{0}^{\infty} b_{e} \mathrm{~d} b_{e} K_{1}\left(m_{e} b_{e}\right) \int_{0}^{\infty} b_{\pi} \mathrm{d} b_{\pi} I\left(b_{\pi}, Q_{\|}\right) \\
& \times 2 \pi \int_{0}^{2 \pi} \mathrm{d} \phi J_{0}(\sqrt{X})\{-\sin \phi\} \exp \left[i \chi_{C}\left(B_{e}\right],\right.
\end{aligned}
$$

with $X$ and $B_{e}$ defined by

$$
\begin{aligned}
X & =\left(q_{\perp} b_{e}\right)^{2}+\left(Q_{\perp} b_{\pi}\right)^{2}-2 q_{\perp} b_{e} Q_{\perp} b_{\pi} \cos \phi, \\
B_{e}^{2} & =b_{e}^{2}+b_{\pi}^{2}+2 b_{e} b_{\pi} \cos \left(\phi+\phi_{q}-\phi_{Q}\right) .
\end{aligned}
$$

For general charge and matter distributions $I\left(b_{\pi}, Q_{\|}\right)$is defined by the integral

$$
I\left(b_{\pi}, Q_{\|}\right)=\frac{-2 \pi i}{Q_{\|}} \int_{-\infty}^{\infty} \mathrm{d} z\left[\hat{\mathbf{b}}_{\pi} \cdot \mathbf{E}_{A}\left(\mathbf{b}_{\pi}, z\right)\right] \exp \left[-i z Q_{\|}-\frac{1}{2} \sigma_{\pi}^{\prime} T\left(b_{\pi}, z\right)\right] .
$$

For a point-charge distribution and neglect of meson rescattering it simplifies to

$$
I_{p c}\left(b_{\pi}, Q_{\|}\right)=K_{1}\left(Q_{\|} b_{\pi}\right),
$$

where for negative values of the argument $K_{1}(-x)=-K_{1}(x)$.

In Born approximation, i.e. for a nucleus point-charge distribution and with neglect of electron and pion distortion, the amplitudes $K(\mathbf{q}, \mathbf{Q})$ and $L(\mathbf{q}, \mathbf{Q})$ reduce to

$$
\begin{aligned}
K_{B}(\mathbf{q}, \mathbf{Q}) & =\frac{q_{\perp}}{\mathbf{q}_{\perp}^{2}+m_{e}^{2}} \cdot \frac{Q_{\perp}}{\mathbf{Q}_{\perp}^{2}+Q_{\|}^{2}}, \\
L_{B}(\mathbf{q}, \mathbf{Q}) & =0
\end{aligned}
$$

with $-Q_{\|}=m_{\pi}^{2} / 2 K_{\|}$. In this case the integrals factorize. In fact, the functional dependence on the angles $\phi_{q}$ and $\phi_{Q}$ factorizes except in the Coulomb-phase-shift function. 


\section{HADRONIC PRODUCTION}

The hadronic contribution represents hadronic interactions between the high-energy photon and the nucleons in the nucleus. Those interactions can be described as exchange interactions where the exchanged particle, as in Fig. 2, is a rho or an omega meson. For omega exchange proton and neutron contributions add whereas for rho exchange they subtract. In addition, the rho interaction is weaker and we shall therefore neglect its contribution. The structure of the omega-exchange contribution is the same as that of photon exchange, except for the replacement of the Coulomb potential by the Yukawa potential. Hence, the nuclear-electric field $\mathbf{E}_{A}(\mathbf{r})$ is replaced by the nuclear-omega field

$$
\mathbf{E}_{\omega}(\mathbf{r})=\nabla_{b} \frac{1}{4 \pi i} \int \mathrm{d}^{3} r^{\prime} \hat{\rho}_{h d}\left(\mathbf{r}^{\prime}\right) \frac{e^{-m_{\omega}\left|\mathbf{r}-\mathbf{r}^{\prime}\right|}}{\left|\mathbf{r}-\mathbf{r}^{\prime}\right|}
$$

where $A \hat{\rho}_{h d}(\mathbf{r})$ is the hadronic density distribution as seen by the omega meson. The omega propagator can be displayed by rewriting this expression as

$$
\mathbf{E}_{\omega}(\mathbf{r})=\frac{1}{i(2 \pi)^{3}} \nabla_{b} \int \mathrm{d}^{3} q e^{-i \mathbf{q} \cdot \mathbf{r}} \frac{1}{\mathbf{q}^{2}+m_{\omega}^{2}} S_{0}(\mathbf{q})
$$

with $S_{0}(\mathbf{q})$ the nuclear form factor,

$$
S_{0}(\mathbf{q})=\int \mathrm{d}^{3} r e^{i \mathbf{q} \cdot \mathbf{r}} \hat{\rho}_{h d}(\mathbf{r})
$$

Unfortunately, high-energy photoproduction of pseudoscalar mesons by nucleons is not well described by the meson exchanges. But Guidal et al. [8] have shown that by Reggeizing those exchanges a good description of data can be obtained. Reggeization means replacing the the omega-pole factor

$$
\mathcal{P}_{\omega}=\frac{1}{t-m_{\omega}^{2}}
$$

by its Reggeized version, which is

$$
\mathcal{P}_{\omega}(s, t)=\left(\frac{s}{s_{0}}\right)^{\alpha_{\omega}(t)-1} \frac{\pi \alpha_{\omega}^{\prime}}{\sin \left(\pi \alpha_{\omega}(t)\right)} \cdot \frac{1}{\Gamma\left(\alpha_{\omega}(t)\right)} \cdot \frac{S_{\omega}+e^{-i \pi \alpha_{\omega}(t)}}{2} .
$$

The signature $S_{\omega}=1$, and the parametrization of the omega trajectory, $\alpha_{\omega}(t)$, has been determined through comparison with photoproduction data [8].

For an ambituous calculation that wants to consider the full Regge structure the definition of the nuclear-omega field becomes

$$
\mathbf{E}_{\omega}(\mathbf{r})=\frac{1}{i(2 \pi)^{3}} \nabla_{b} \int \mathrm{d}^{3} q e^{-i \mathbf{q} \cdot \mathbf{r}} \frac{1}{m_{\omega}^{2}} S_{0}(\mathbf{q}) \mathcal{P}_{\omega}\left(\mathbf{q}^{2}\right) / \mathcal{P}_{\omega}\left(\mathbf{q}^{2}=0\right)
$$


with $S_{0}(\mathbf{q})$ the nuclear form factor of Eq.(38). There could also be other form factors besides the Regge factor. The $\mathbf{q}^{2}$-dependence of the Regge factor is much weaker than that of the nuclear factor. Neglecting it altogether leads to a simple expresion for the nuclear-omega field,

$$
\mathbf{E}_{\omega}(\mathbf{r})=\frac{1}{i m_{\omega}^{2}} \nabla_{b} \hat{\rho}_{h d}(\mathbf{r}) .
$$

The omega-exchange amplitude is decomposed as follows;

$$
\begin{aligned}
\mathcal{M}_{\omega} & =-i \mathcal{N}_{\omega}\left[G_{\omega}(\mathbf{q}, \mathbf{Q})-H_{\omega}(\mathbf{q}, \mathbf{Q}) i \boldsymbol{\sigma} \cdot \hat{k}_{1}\right] \\
\mathcal{N}_{\omega} & =A \frac{\alpha g_{\omega \pi \gamma} g_{\omega N N}}{4 \pi m_{\pi}} m_{\omega}^{2} \mathcal{P}_{\omega}(0) .
\end{aligned}
$$

Pure omega exchange corresponds to $m_{\omega}^{2} \mathcal{P}_{\omega}(0)=-1$. The functions of Eq.(43) are defined in complete analogy with the two-photon exchange amplitudes,

$$
\begin{aligned}
G_{\omega}(\mathbf{q}, \mathbf{Q})= & \int \mathrm{d}^{3} r_{e} \int \mathrm{d}^{3} r_{\pi} e^{i \mathbf{q} \cdot\left(\mathbf{r}_{e}-\mathbf{r}_{\pi}\right)} e^{-i \mathbf{Q} \cdot \mathbf{r}_{\pi}} \exp \left[i \chi_{C}\left(b_{e}\right)-\frac{1}{2} \sigma_{\pi}^{\prime} T\left(\mathbf{b}_{\pi}, z_{\pi}\right)\right] \\
& \times\left[\mathbf{E}_{e}\left(\mathbf{r}_{e}-\mathbf{r}_{\pi}\right) \times \mathbf{E}_{\omega}\left(\mathbf{r}_{\pi}\right)\right] \cdot \hat{k}_{1}, \\
H_{\omega}(\mathbf{q}, \mathbf{Q})= & \int \mathrm{d}^{3} r_{e} \int \mathrm{d}^{3} r_{\pi} e^{i \mathbf{q} \cdot\left(\mathbf{r}_{e}-\mathbf{r}_{\pi}\right)} e^{-i \mathbf{Q} \cdot \mathbf{r}_{\pi}} \exp \left[i \chi_{C}\left(b_{e}\right)-\frac{1}{2} \sigma_{\pi}^{\prime} T\left(\mathbf{b}_{\pi}, z_{\pi}\right)\right] \\
& \times\left[\mathbf{E}_{e}\left(\mathbf{r}_{e}-\mathbf{r}_{\pi}\right) \cdot \mathbf{E}_{\omega}\left(\mathbf{r}_{\pi}\right)\right] .
\end{aligned}
$$

The definitions of $G_{\omega}(\mathbf{q}, \mathbf{Q})$ and $H_{\omega}(\mathbf{q}, \mathbf{Q})$ in Eqs (45) and (46) parallel the definitions of $G(\mathbf{q}, \mathbf{Q})$ and $H(\mathbf{q}, \mathbf{Q})$ in Eqs (17) and (18). To get from the latter to the former we replace $\mathbf{E}_{A}\left(\mathbf{r}_{\pi}\right)$ by $\mathbf{E}_{\omega}\left(\mathbf{r}_{\pi}\right)$. In exactly the same way we define $K_{\omega}(\mathbf{q}, \mathbf{Q})$ and $L_{\omega}(\mathbf{q}, \mathbf{Q})$ to get the decomposition

$$
\begin{aligned}
& G_{\omega}(\mathbf{q}, \mathbf{Q})=K_{\omega}(\mathbf{q}, \mathbf{Q})\left(\hat{\mathbf{q}}_{\perp} \times \hat{\mathbf{Q}}_{\perp}\right) \cdot \hat{k}_{1}+L_{\omega}(\mathbf{q}, \mathbf{Q}) \hat{\mathbf{q}}_{\perp} \cdot \hat{\mathbf{Q}}_{\perp} \\
& H_{\omega}(\mathbf{q}, \mathbf{Q})=K_{\omega}(\mathbf{q}, \mathbf{Q}) \hat{\mathbf{q}}_{\perp} \cdot \hat{\mathbf{Q}}_{\perp}-L_{\omega}(\mathbf{q}, \mathbf{Q})\left(\hat{\mathbf{q}}_{\perp} \times \hat{\mathbf{Q}}_{\perp}\right) \cdot \hat{k}_{1} .
\end{aligned}
$$

However, the replacement of $\mathbf{E}_{A}\left(\mathbf{r}_{\pi}\right)$ by $\mathbf{E}_{\omega}\left(\mathbf{r}_{\pi}\right)$ makes the structure functions connected with omega exchange quite different from those connected with photon exchange. In general, $L_{\omega}(\mathbf{q}, \mathbf{Q})$ is small and may be neglected. For more details see the Appendix. 


\section{FACTORIZATION}

The formulae given so far apply to arbitrary nuclei and arbitrary momentum transfers $\mathbf{Q}_{\perp}$ and $\mathbf{q}_{\perp}$, as long as they remain much smaller than the longitudinal momenta $k_{1}$ and $K$. The tricky point in their evaluation is that the integrations over $\mathbf{r}_{e}$ and $\mathbf{r}_{\pi}$ are intertwined, as the electron interacts with both nucleus and meson. However, in some circumstancies the integrations factorize.

Consider production of eta mesons at $11 \mathrm{GeV}$, and start with the Coulomb terms $K(\mathbf{q}, \mathbf{Q})$ and $L(\mathbf{q}, \mathbf{Q})$ of Eqs (28) and (29) . The cut-off in the $b_{e}$ integration is set by the inverse of the electron mass $1 / m_{e} \approx 390 \mathrm{fm}$, and the cut-off in the $b_{\eta}$ integration by the inverse of of the longitudinal momentum transfer $2 K_{\|} / m_{\eta}^{2} \approx 14 \mathrm{fm}$. The only coupling between the $b_{e}$ and $b_{\eta}$ dependencies is in the argument, Eq.(31), of the Coulomb-phase function. In view of the small ovelap region, we may here neglect the dependence on $b_{\eta}$. As a result, the integrands of Eqs (28) and (29) factorize, and the function $L(\mathbf{q}, \mathbf{Q})$ vanishes after integration over the $\phi$ variable. These arguments are weakened when we consider momentum transfers $\mathbf{q}_{\perp}$ so large that there are strong oscillations in the $b_{e}$ integrand. Then the main contributions to the Coulomb integral come from regions much closer to the nucleus.

In the factorized approximation

$$
\begin{aligned}
L(\mathbf{q}, \mathbf{Q}) & =0 \\
K(\mathbf{q}, \mathbf{Q}) & =F_{C}(\mathbf{q}) F_{A}(\mathbf{Q})
\end{aligned}
$$

with the functions $F_{C}(\mathbf{q})$ and $F_{A}(\mathbf{Q})$ defined by

$$
\begin{aligned}
& F_{C}(\mathbf{q})=\int \mathrm{d}^{3} r_{e} e^{i\left[\mathbf{q} \cdot \mathbf{r}_{e}+\chi_{C}\left(b_{e}\right)\right]} \hat{\mathbf{q}}_{\perp} \cdot \mathbf{E}_{e}\left(\mathbf{r}_{e}\right) \\
& F_{A}(\mathbf{Q})=\int \mathrm{d}^{3} r_{\eta} e^{-i \mathbf{Q} \cdot \mathbf{r}_{\eta}} \hat{\mathbf{Q}}_{\perp} \cdot \mathbf{E}_{A}\left(\mathbf{r}_{\eta}\right) \exp \left[-\frac{1}{2} \sigma_{\eta}^{\prime} T\left(\mathbf{b}_{\eta}, z_{\eta}\right)\right] .
\end{aligned}
$$

The function $F_{C}(\mathbf{q})$ describes Coulomb scattering of the electron, and for a nuclear-pointcharge distribution it can be integrated exactly, Ref.[3, 9]. The $F_{A}(\mathbf{Q})$ is the Coulomb contribution (one-photon exchange) to eta-nucleus photoproduction.

In the hadronic contribution the integration over $b_{\eta}$ is limited to the nuclear region. Thus, in this case the integrals always factorize, so that $L_{\omega}(\mathbf{q}, \mathbf{Q})=0$ and

$$
\begin{aligned}
K_{\omega}(\mathbf{q}, \mathbf{Q}) & =F_{C}(\mathbf{q}) F_{\omega}(\mathbf{Q}) \\
F_{\omega}(\mathbf{Q}) & =\int \mathrm{d}^{3} r_{\eta} e^{-i \mathbf{Q} \cdot \mathbf{r}_{\pi}} \hat{\mathbf{Q}}_{\perp} \cdot \mathbf{E}_{\omega}\left(\mathbf{r}_{\eta}\right) \exp \left[-\frac{1}{2} \sigma_{\eta}^{\prime} T\left(\mathbf{b}_{\eta}, z_{\eta}\right)\right]
\end{aligned}
$$


The sum of Coulomb and hadronic contributions can be written as

$$
G(\mathbf{q}, \mathbf{Q})+G_{\omega}(\mathbf{q}, \mathbf{Q})=\sum_{\lambda}\left[\hat{\mathbf{q}}_{\perp} \cdot \mathbf{e}_{\lambda} F_{C}(\mathbf{q})\right]\left[\left(\hat{\mathbf{Q}}_{\perp} \times \hat{k}_{1}\right) \cdot \mathbf{e}_{\lambda}\left\{F_{A}(\mathbf{Q})+F_{\omega}(\mathbf{Q})\right\}\right]
$$

where the sum runs over the two photon polarization vectors orthogonal to $\hat{k}_{1}$, or $\hat{q}$. For the sum $H(\mathbf{q}, \mathbf{Q})+H_{\omega}(\mathbf{q}, \mathbf{Q})$ the expression is the same but with $\hat{\mathbf{q}}_{\perp} \cdot \mathbf{e}_{\lambda}$ replaced by $\left(\hat{\mathbf{q}}_{\perp} \times \hat{k}_{1}\right) \cdot \mathbf{e}_{\lambda}$.

The two terms in the last bracket of Eq.(55) represent the eta-nucleus photoproduction amplitude initiated by photons of polarization $\mathbf{e}_{\lambda}$. The first term $F_{A}(\mathbf{Q})$ is the Coulombphotoproduction amplitude, the second term $F_{\omega}(\mathbf{Q})$ the hadronic-photoproduction amplitude. It is also important to note that the photons radiated by the electron are transverse photons, not Coulomb photons.

In pion-electroproduction the hadronic amplitudes factorize, but the Coulomb amplitudes do not. Hence, the pion-electroproduction amplitude will not have a decomposition as in Eq.(55). 


\section{SHADOWING}

The hadronic contribution is modified by shadowing, a multple scattering contribution where the initial photon is first converted into a rho meson, which in a subsequent collision with another nucleon creates the pion. This phenomenon is described in detail in Ref.[10].

In the hadronic term the high-energy photon produces the final-state pion through omegameson exchange with a single nucleon. The correspondin amplitude is proportional to $f_{\omega}(\gamma N \rightarrow \pi N)$. In the shadowing term the high-energy photon first creates a rho meson by diffractive production on a nucleon. This step is proportional to $f_{P}(\gamma N \rightarrow \rho N)$. In the second step the rho meson collides with another nucleon creating a pi meson through omega-meson exchange. This step is proportional to the amplitude $f_{\omega}(\rho N \rightarrow \pi N)$. Now, if the hadronic interaction of photons proceeds via the rho meson, we expect the relation

$$
f_{P}(\gamma N \rightarrow \rho N) f_{\omega}(\rho N \rightarrow \pi N)=f_{P}(\rho N \rightarrow \rho N) f_{\omega}(\gamma N \rightarrow \pi N)
$$

where $f_{P}(\rho N \rightarrow \rho N)$ is the diffractive rho-nucleon-scattering amplitude. We remark that the rho meson is off its mass shell in two of the amplitudes, one on each side. It is assumed that the off-shell factors cancel out.

The amplitude relation (56) leads to a replacement of the omega field, Eq.(42), by

$$
\mathbf{E}_{\omega}(\mathbf{r})=\frac{1}{i m_{\omega}^{2}} \nabla_{b} \hat{\rho}_{h d}(\mathbf{r})\left\{1-\frac{1}{2} \sigma_{\rho}^{\prime} \int_{-\infty}^{z} \mathrm{~d} z^{\prime} n\left(\mathbf{b}, z^{\prime}\right) e^{i \Delta_{\rho}\left(z^{\prime}-z\right)} \exp \left[-\frac{1}{2} \sigma_{\rho}^{\prime} \int_{z^{\prime}}^{z} \mathrm{~d} z^{\prime \prime} n\left(\mathbf{b}, z^{\prime \prime}\right)\right]\right\}
$$

with $n(\mathbf{r})=A \hat{\rho}_{h d}(\mathbf{r})$. The second term inside the brackets is the shadowing term. The intermediate rho meson is produced at $z^{\prime}$ and the final-state pion at $z$. Between these two points, the distortion of the wave is due rho-meson scattering. The longitudinal momentum transfer in the $\gamma N \rightarrow \rho N$ reaction at $z^{\prime}$ is $\Delta_{\rho}=m_{\rho}^{2} / 2 K_{\|}$. The longitudinal momentum transfer in the $\rho N \rightarrow \pi N$ reaction at $z$ is $\Delta_{\pi}=\left(m_{\pi}^{2}-m_{\rho}^{2}\right) / 2 K_{\|}=-\Delta_{\rho}-Q_{\|}$. In the direct hadronic term the longitudinal momentum transfer to the pion is $-Q_{\|}=m_{\pi}^{2} / 2 K_{\|}$. This phase factor is outside the omega field $\mathbf{E}_{\omega}(\mathbf{r})$. So is the pion distortion, Eqs (47) and (48). 


\section{CROSS-SECTION DISTRIBUTIONS}

The unpolarized-cross-section distribution as derived in Ref.[3] reads

$\frac{\mathrm{d} \sigma}{\mathrm{d}^{2} k_{2 \perp} \mathrm{d}^{2} K_{\perp} \mathrm{d} k_{2 \|}}=\frac{1}{\pi K_{\|}}\left(\frac{Z \alpha^{2} g_{\pi \gamma \gamma}}{m_{\pi}}\right)^{2}\left[\left|K(\mathbf{q}, \mathbf{Q})+\mathcal{R} K_{\omega}(\mathbf{q}, \mathbf{Q})\right|^{2}+\left|L(\mathbf{q}, \mathbf{Q})+\mathcal{R} L_{\omega}(\mathbf{q}, \mathbf{Q})\right|^{2}\right]$

with $\mathcal{R}$ the ratio of coupling constants,

$$
\mathcal{R}=-\mathcal{N}_{\omega} / \mathcal{N}_{2 \gamma}
$$

The parameter $\mathcal{R}$ depends only weakly on atomic number so we do not gain relative strength for the Coulomb term by going to heavier nuclei. The advantage is instead that the crosssection values themselves grow as $Z^{2}$. An interesting feature of Eq.(58) is that in the unpolarized cross-section distributions $K$ - and $L$-amplitudes do not interfere. The sign of $\mathcal{R}$ must be determined by experiment.

The transverse momenta in Eq.(58) are restricted to the regions $q_{\perp}, Q_{\perp} \ll k_{1}, k_{2}, K$. For cross-section distributions such that $Q_{\perp} R_{u} \approx 1$, nuclear structure becomes important and numerical evaluation necessary. We have also stressed that depending on the value of $q_{\perp} / m_{e}$ integrals may or may not factorize. The complicated functional dependences make a general overview difficult. For this reason we concentrate on production of pions and etas in the Coulomb region, which is of special importance to the PrimEx experiment.

Pi-meson production.

Pion electroproduction at $11 \mathrm{GeV} / c$ was investigated in Ref.[3], with emphasis on the double-Coulomb region, where $Q_{\perp} \approx\left|Q_{\|}\right|=0.85 \mathrm{MeV} / c$ and $q_{\perp} \approx m_{e}=0.52 \mathrm{MeV} / c$. In this particular case there is, in the Coulomb amplitude, a strong overlap between the impactparameter domains of pion and electron. As a consequence the electroproduction amplitude does not factorize and the predictions differ considerably from the Born approximation. Non-factorization means that the pion-nucleus-photoproduction cross section is not a factor of the pion-nucleus-electroproduction cross section.

However, non-factorization does not mean we cannot determine the pion-decay constant $g_{\pi \gamma \gamma}$. We can, as long as we have a reliable theory for the cross-section distribution and as long as the hadronic contribution is much smaller than the Coulomb contribution.

In Ref.[3] the relative size of Coulomb and hadronic amplitudes was estimated. Here, we assume pure omega exchange and neglect electron and pion distortions. Then, at the double 
peak, the ratio of Coulomb to hadronic amplitude strengths becomes

$$
R_{\pi}=\left[\frac{Z \alpha^{2} g_{\pi \gamma \gamma}}{m_{\pi}} \frac{1}{2 Q_{\|}^{2}}\right] /\left[\frac{A \alpha g_{\omega \pi \gamma} g_{\omega N N}}{4 \pi m_{\pi}} \frac{1}{m_{\omega}^{2}}\right]=110 .
$$

The numerical value refers to lead nuclei. Taking Regge exchange instead of omega exchange implies dividing by $\left|\mathcal{P}_{\omega}(0) m_{\omega}^{2}\right|=0.123$ giving a new value, $R_{\pi}=910$. With such a large ratio it should be easy to isolate the Coulomb contribution from the hadronic background, making a determination of $g_{\pi \gamma \gamma}$ realistic.

Photoproduction of pseudoscalar mesons by protons, including the Coulomb term, was investigated in Ref.[11], and photoproduction by nuclei most recently in Refs [12, 13]. Eta-meson production.

Production of eta mesons is at $11 \mathrm{GeV} / c$ simpler to calculate than production of pi mesons. The double-Coulomb region is now the region where $Q_{\perp} \approx\left|Q_{\|}\right|=8.5 \mathrm{MeV} / \mathrm{c}$, and $q_{\perp} \approx m_{e}=0.52 \mathrm{MeV} / c$. Consequently, overlap between the integration domains of the etaand electron-impact-parameter variables is small. The factorization discussed in Sect. $\mathrm{V}$ applies and the cross-section distribution contains the factor

$$
\left|F_{C}(\mathbf{q})\right|^{2}\left|F_{A}(\mathbf{Q})+F_{\omega}(\mathbf{Q})\right|^{2}
$$

The first factor in Eq. (61) is the squared Coulomb amplitude of the electron. Its analytic form is given in Refs [3, 9]. It is not the usual Coulomb-scattering amplitude since it describes exchange of transverse photons, not Coulomb photons. In the Coulomb factor $F_{C}(\mathbf{q})$ the longitudinal momentum $q_{\|}$enters in the combination $q_{\|} / \gamma \approx m_{e}$. This factor exhibits a Primakoff-peak structure in the variable $q_{\perp}$ with a peak value at $q_{\perp} \approx m_{e}$.

The second factor in Eq.(61) is the squared eta-nucleus-photoproduction amplitude. This amplitude has two parts; the Coulomb amplitude $F_{A}(\mathbf{Q})$ and the hadronic amplitude $F_{\omega}(\mathbf{Q})$. Assuming omega exchange and neglecting electron and pion rescattering the ratio $R_{\eta}$ of Coulomb to hadronic amplitudes is algebraically the same as the ratio for pions, Eq.(60), but with all pi indices replaced by eta indices. For lead nuclei $R_{\eta}=1.8$ with omega exchange and $R_{\eta}=15$ with Regge exchange. The Coulomb amplitude dominates but only weakly. For an accurate determination of $g_{\eta \gamma \gamma}$ higher energies are needed.

Eta-prime-meson production.

At $11 \mathrm{GeV} / c$ the double-Coulomb region in eta-prime production is the region where $Q_{\perp} \approx\left|Q_{\|}\right|=43 \mathrm{MeV} / c$, and $q_{\perp} \approx m_{e}=0.52 \mathrm{MeV} / c$. As for eta-meson production 
the factorization discussed in Sect. $\nabla$ applies as well as Eq.(61). The second factor in Eq.(61) is now the squared eta-prime-nucleus-photoproduction amplitude. Assuming omega exchange and neglecting electron and pion rescattering the ratio $R_{\eta^{\prime}}$ of Coulomb to hadronic amplitudes is algebraically the same as the ratio for pions, Eq.(60), but with all pi indices replaced by eta-prime indices. For lead nuclei $R_{\eta^{\prime}}=0.30$ with omega exchange and $R_{\eta^{\prime}}=2.5$ with Regge exchange. Consequently, a determination of $g_{\eta^{\prime} \gamma \gamma}$ is not possible at this energy. 


\section{APPENDIX}

Define the normalized Regge propagator as $\hat{\mathcal{P}}_{\omega}\left(\mathbf{q}^{2}\right)=\mathcal{P}_{\omega}\left(\mathbf{q}^{2}\right) / \mathcal{P}_{\omega}\left(\mathbf{q}^{2}=0\right)$. With this definition we can write the nuclear-omega field of Eq.(41) as

$$
\begin{aligned}
\mathbf{E}_{\omega}(\mathbf{r}) & =\frac{1}{i(2 \pi)^{3} m_{\omega}^{2}} \nabla_{b} \int \mathrm{d}^{3} q e^{-i \mathbf{q} \cdot \mathbf{r}} S_{0}\left(\mathbf{q}^{2}\right) \hat{\mathcal{P}}_{\omega}\left(\mathbf{q}^{2}\right) \\
& =\frac{i}{2 \pi^{2} m_{\omega}^{2}} \frac{\mathbf{b}}{r} \int_{0}^{\infty} q^{3} \mathrm{~d} q j_{1}(q r) S_{0}\left(\mathbf{q}^{2}\right) \hat{\mathcal{P}}_{\omega}\left(\mathbf{q}^{2}\right)
\end{aligned}
$$

If we like the coordinate-space description better we introduce

$$
\hat{\mathcal{P}}_{\omega}(\mathbf{r})=\frac{1}{(2 \pi)^{3}} \int \mathrm{d}^{3} q e^{-i \mathbf{q} \cdot \mathbf{r}} \hat{\mathcal{P}}_{\omega}(\mathbf{r})
$$

and get

$$
\mathbf{E}_{\omega}(\mathbf{r})=\frac{1}{i m_{\omega}^{2}} \nabla_{b} \int \mathrm{d}^{3} r^{\prime} \hat{\rho}_{h d}\left(\mathbf{r}-\mathbf{r}^{\prime}\right) \hat{\mathcal{P}}_{\omega}\left(\mathbf{r}^{\prime}\right) .
$$

A simplification occurs if we put $\hat{\mathcal{P}}_{\omega}\left(\mathbf{q}^{2}\right)=1$, which means neglecting the angular variation of the Regge factor as compared with the nuclear form factor. In this approximation $\hat{\mathcal{P}}_{\omega}(\mathbf{r})=$ $\delta(\mathbf{r})$, and we recover Eq.(42).

In momentum space the omega field becomes

$$
\mathbf{E}_{\omega}(\mathbf{Q})=\int \mathrm{d}^{3} r e^{-i \mathbf{Q} \cdot \mathbf{r}} \mathbf{E}_{\omega}(\mathbf{r})=\frac{\mathbf{Q}_{\perp}}{m_{\omega}^{2}} S_{0}(\mathbf{Q})
$$

for the simplified Regge case, $\hat{\mathcal{P}}_{\omega}(\mathbf{r})=\delta(\mathbf{r})$.

We end with an alternative method for deriving Eqs (26, 27). Decompose the unit vector $\hat{b}_{e}$ along the unit vector $\hat{q}$, which here stands for the impact-plane component of $\boldsymbol{q}$, i.e.

$$
\begin{aligned}
\hat{b}_{e} & =\left[\hat{b}_{e} \cdot \hat{q}\right] \hat{q}+\left[\hat{b}_{e} \cdot(\hat{k} \times \hat{q})\right] \hat{k} \times \hat{q} \\
& =\cos \left(\varphi_{e}\right) \hat{q}+\sin \left(\varphi_{e}\right) \hat{k} \times \hat{q} .
\end{aligned}
$$

We perform the same decomposition for $\hat{b}_{\pi}$ but along $\hat{Q}$ and with angle $\varphi_{\pi}$. This gives

$$
\begin{aligned}
\hat{b}_{e} \cdot \hat{b}_{\pi} & =\cos \varphi \hat{q} \cdot \hat{Q}+\sin \varphi(\hat{q} \times \hat{Q}) \cdot \hat{k} \\
\left(\hat{b}_{e} \times \hat{b}_{\pi}\right) \cdot \hat{k} & =-\sin \varphi \hat{q} \cdot \hat{Q}+\cos \varphi(\hat{q} \times \hat{Q}) \cdot \hat{k}
\end{aligned}
$$

with $\varphi=\varphi_{e}-\varphi_{\pi}$. 
[1] E. Hadjimichael and S. Fallieros, Phys. Rev. C 39, 1438 (1989).

[2] R.J. Glauber, in Lectures in theoretical physics, edited by W.E. Brittin and L.G. Dunham (Interscience, New York, 1959), vol. 1, p. 315; in High-Energy Physics and Nuclear Structure, edited by S. Devons (Plenum, New York, 1970), p. 207.

[3] G. Fäldt, Phys. Rev. C83, 044606 (2011).

[4] A. Gasparian et al., Proposal E-02-103 (2001).

[5] G. Fäldt, Nucl. Phys. B 43, 591 (1972).

[6] L. Landau et E. Lifchitz, Théorie des champs (Éditions Mir, Moscou 1970).

[7] G. Fäldt, Phys. Rev. D2, 846 (1970).

[8] M. Guidal, J.-M. Laget, and M. Vanderhaegen, Nucl. Phys. A 627, 645 (1997).

[9] G. Fäldt and U. Tengblad, Phys. Rev. C 79, 014607 (2009); G. Fäldt, Phys. Rev. C 82, $037603(2010)$.

[10] D.R. Yennie, in Hadronic Interactions of Electrons and Photons, edited by J. Cumming and H. Osborn (Academic Press, New York, 1971), p. 321.

[11] J.-M. Laget, Phys. Rev. C 72, 022202(R) (2005).

[12] S. Gevorkian et al., Phys. Rev. C 80, 055201 (2009).

[13] M.M. Kaskulov and U. Mosel, arXiv:1103.2097. 